\title{
Advances in mobile communications and computing
}

\author{
Arjan Durresi $^{\mathrm{a}}$ and Mieso Denko ${ }^{\mathrm{b}}$

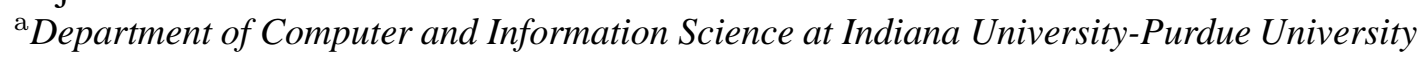 \\ Indianapolis, 723 W. Michigan St., Indianapolis, IN 46202, USA \\ E-mail:durresi@cs.iupui.edu \\ ${ }^{\mathrm{b}}$ Department of Computing and Information Science, Guelph, Ontario, Canada, N1G 2W1 \\ E-mail:denko@cis.uoguelph.ca
}

Advances in technology have enabled the combinations of mobile and ubiquitous computing to be increasingly present in our daily life. For example, progress in low power wireless communications along with silicon integration of various functionalities, such as sensing, communications, intelligence and actuations are emerging as a critically important disruptive computer class based on a new platform, networking structure and interface that enable novel, low cost, high volume applications such as nuclear, biological and chemical attack detection and protection, home automation, battlefield surveillance and environmental monitoring. To fulfill the potential for new services, applications and businesses offered by new computing and communication capabilities, several complex research issues need to be further studied and resolved.

This special issue highlights advances in various aspects of mobile communications and computing, and is organized mainly from the papers of the The 22nd IEEE International Conference on Advanced Information Networking and Applications AINA 2008, held in GinoWan, Okinawa, Japan, March 25-28, 2008. The conference received 469 submissions and every paper was reviewed carefully by 3 reviewers. Based on their quality and significance 146 papers were accepted in AINA 2008. We received 19 papers for this special issue. After two more rounds of review, we accepted 6 papers based on their quality and suitability to the special issue as well as the journal.

Security is the major requirement of mobile and e-commerce systems. These systems and environments need many distributed access points, for the creation and management of secure identities and for the secure recognition of users. Traditionally, these access points can be made possible by a software system with a main central server. In the first paper, Conti et al. propose the study and implementation of a multimodal technique, based on biometric information, for identity management and personal ubiquitous authentication.

In the second paper, Santangelo et al. describe a framework aimed at streamlining the design process of multi-channel, multimodal interfaces enabling full reuse of software components in mobile systems. This framework is called the eXtensible Presentation architecture and Language (XPL), a presentation language based on design pattern paradigm that keeps separated the presentation layer from the underlying 
programming logic. The language supplies a methodology to expedite multimodal interface development and to reduce the effort to implement interfaces for multiple access devices, by means of using the same code.

In the next paper, Izumi et al. propose a supervisory system, uEyes, that considers actual situations and social aspects of users in a ubiquitous computing environment. Using Social Context Awareness, uEyes can combine environmental information acquired from sensors in the real world and common-sense knowledge related to human activities in daily life.

In the forth paper, Ikeda et al. evaluate the performance of Optimized Link State Routing (OLSR) protocol by experimental and simulation results. The experiments are carried out by using AN implemented testbed and the simulations by using ns- 2 simulator. They also designed and implemented a new interface for the ad-hoc network testbed in order to make easier the experiments.

Cognitive radio technology necessitates accurate and timely sensing of primary users' activity on the chosen set of channels. The simplest selection procedure is a simple random choice of channels to be sensed, but the impact of sensing errors with respect to primary user activity or inactivity differs considerably. In order to improve sensing accuracy and increase the likelihood of finding channels which are free from primary user activity, the selection procedure is modified by assigning different sensing probabilities to active and inactive channels. Vojislav and Jelena Mišić present a probabilistic analysis of this policy and investigate the range of values in which the modulation of sensing probability is capable of maintaining an accurate view of the status of the working channel set. They also present a modification of the probability modulation algorithm that allows for even greater reduction of sensing error in a limited range of the duty cycle of primary users' activity. Finally, they give some guidelines as to the optimum application ranges for the original and modified algorithm, respectively.

In the last paper, Kumar and Park present a model for two associated Poisson processes, through which sensors are distributed in a plane. A probability condition is established for data redundancy among closely located sensor nodes. The model generates a spatial bivariate Poisson process whose parameters depend on the parameters of the two individual Poisson processes and on the distance between the associated points. The proposed model helps in building efficient algorithms for data dissemination in the sensor network. A numerical example is provided investigating the advantage of this model.

As we conclude this overview, we would like to thank all the authors for submitting their papers, and greatly thank many reviewers for their good work to make possible the publication of this special issue on time. In particular, we would like to address our special thanks to Dr. David Taniar for his strong encouragement and support for this Special Issue.

Arjan Durresi and Mieso Denko

Guest Editors

January, 2009

\footnotetext{
Arjan Durresi received his B.E., MSc. and Ph.D. (all summa cum laude) in Electronic-Telecommunications, and a Diploma of Superior Specialization in Telecommunications from La Sapienza University in Rome, Italy and Italian Telecommunications Institute. Since August 2007, Dr. Durresi has been as an Associate Professor with the Department of Computer and Information Science, at Indiana University-Purdue University Indianapolis, IN, USA. Previously he has been with the Department of Computer Science at Louisiana State University, Department of Computer and Information Science in the Ohio State University, Telesoft S.p.A, Rome, Italy and Polytechnic University of Tirana. Dr. Durresi is a Professor of University in Albania, and an Associate Member of Albania Academy of Science.

Dr. Durresi's current research interests include network architectures, heterogeneous wireless networks, security, QoS, routing protocols, traffic management, optical and satellite networks, multimedia networking, performance testing, and bioinformatics. Dr. Durresi has published more than seventy articles in journals and one hundred articles in proceedings of referred international
} 
conference. He is an area editor for the Ad Hoc Networks Journal (Elsevier), Journal of Network and Computer Applications(Elsevier), Journal of Ubiquitous Computing and Intelligence -JUCI, (American Scientific Publishers - ASP), and guest editor for the various International Journals. He is the founder of the IEEE International Workshops on Heterogeneous Wireless Networks - HWISE, and Co-Chair from 2005 to 2009. He is the Co-founder of the International Workshop on Advances in Information Security - WAIS and Co-Chair from 2007 to 2009. He is Co-Chair of AINA2009, Program Vice Chair of ICPADS 2005. Dr. Durresi is a Yamacraw Distinguished Speaker (2003) and a keynote speaker of IEEE AINA 2007. For more information, please visit www.cs.iupui.edu/ durresi.

Mieso Denko received his MSc degree from the University of Wales, UK, and his PhD degree from the University of Natal, South Africa, both in Computer Science. Since November 2002, Dr. Denko has been with the Department of Computing and Information Science, Guelph, Ontario, Canada, where he is now an Associate Professor with Tenure. His current research interests include wireless networks, mobile and pervasive computing, wireless mesh networks, wireless sensor networks and network security.

Dr. Denko is a founder/co-founder of a number of ongoing international workshops and served as program chair/co-chair of a number international conferences. Currently he is serving as guest co-editing of Special Issues for a number of journals including the ACM/Springer Mobile Networks and Applications (MONET) and IEEE Systems journal. Dr. Denko has coedited two books in the areas of pervasive computing and wireless networks, and currently co-editing a forthcoming book on autonomic computing and networking to be published by Springer in April 2009. He is Editorial board member of the Journal of Information Technology Research (JITR), IGI Publishing (2007-), International Journal of Smart Homes (IJSH), Editorial Advisory Board member of the Journal of Ubiquitous Computing and Communications, (UBICC), 2008-, and Associate Editor of the International Journal of Multimedia and Ubiquitous Engineering (IJMUE), SERSC (2008-), International Journal of Communication Systems (IJCS), Wiley, 2008- and Security and Communications Network (SCN), Wiley, 2009-. He is a senior member of the ACM and IEEE. For detailed information, please visit www.cis.uoguelph.ca/ denko 

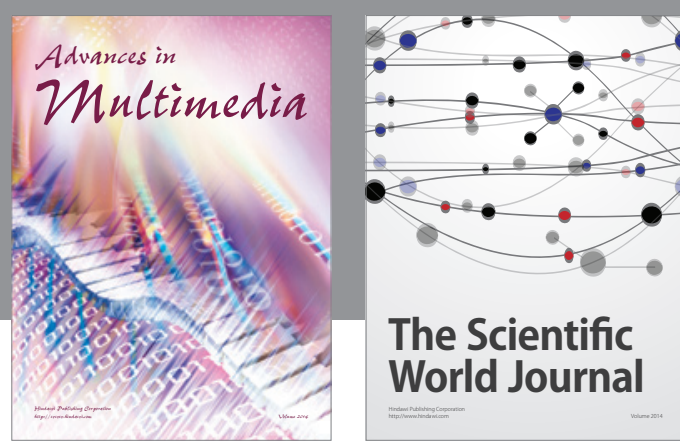

The Scientific World Journal
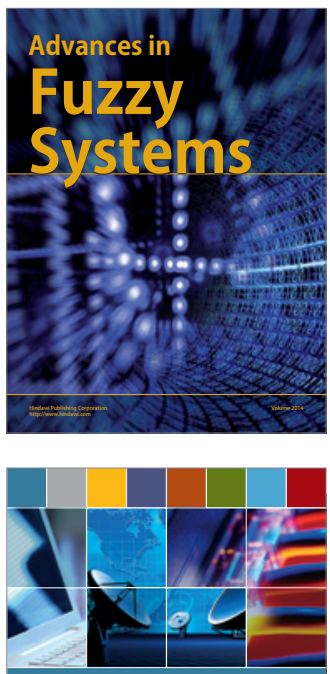

Computer Networks and Communications
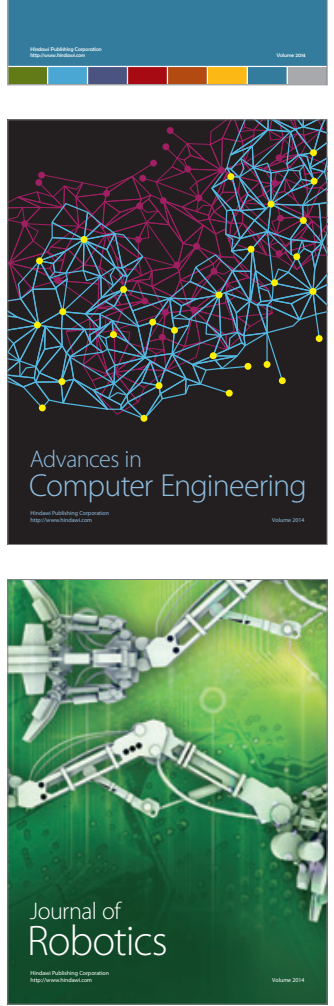
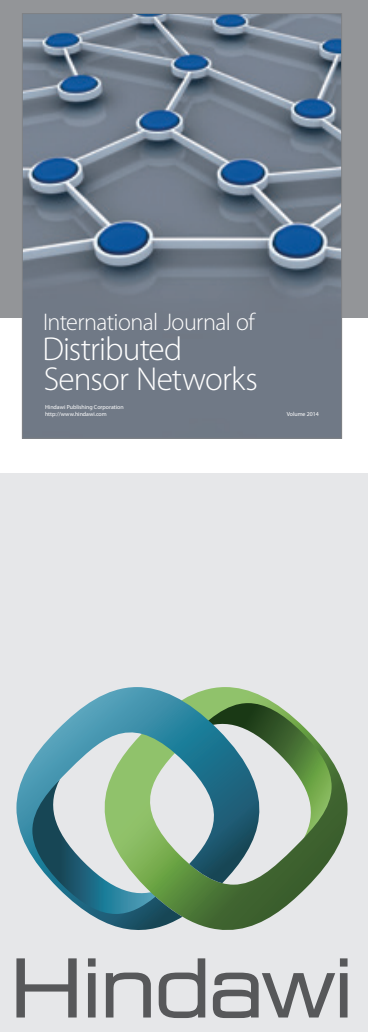

Submit your manuscripts at

http://www.hindawi.com
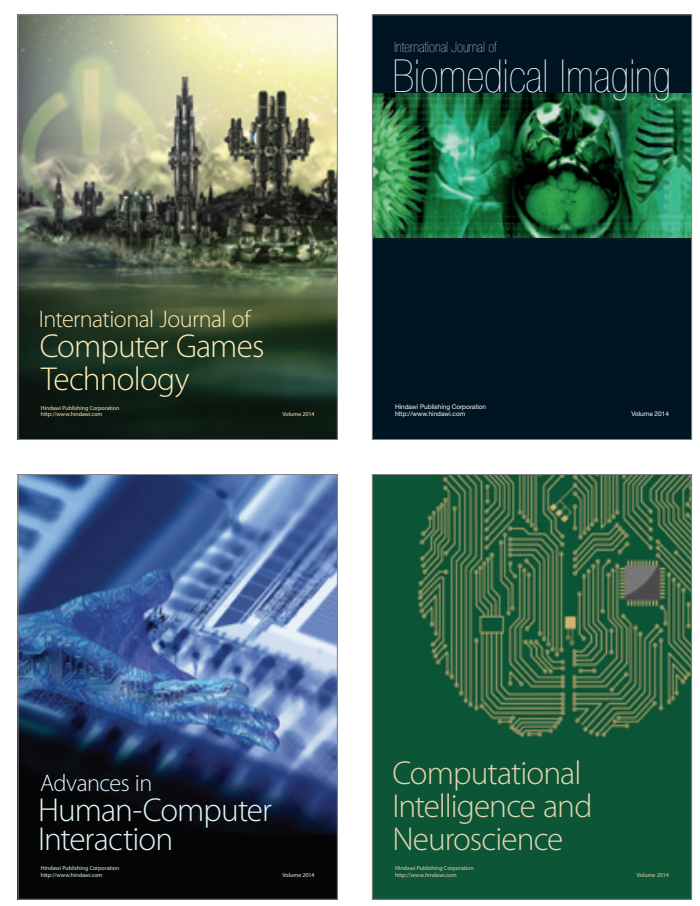
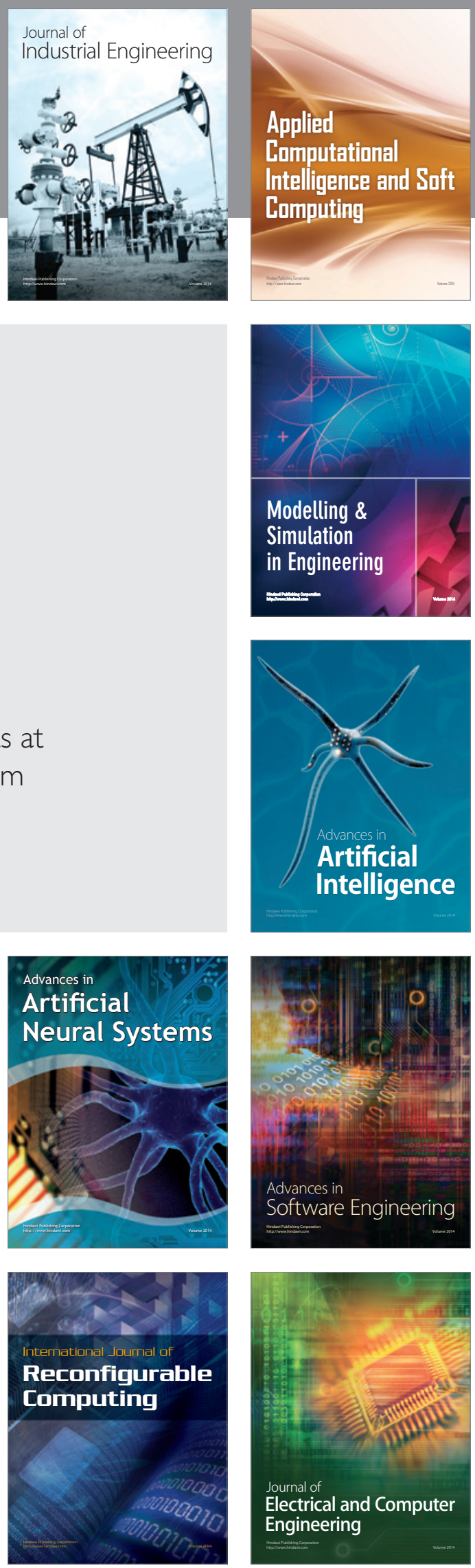